\title{
Avaliação da estrutura da paisagem visando à conservação da biodiversidade em paisagem urbanizada
}

\author{
Landscape structure aiming at the biodiversity conservation of urbanized \\ landscape
}

\author{
Marina Pannunzio Ribeiro ${ }^{\mathrm{I}}$, Kaline de Mello" ${ }^{\mathrm{II}}$, Roberta Averna Valente ${ }^{\mathrm{III}}$
}

\begin{abstract}
Resumo
Considerando que a expansão urbana e o aumento das atividades antrópicas geram perda e fragmentação dos hábitats naturais, ameaçando a biodiversidade, a conservação dos remanescentes florestais urbanos é de extrema importância para a persistência de espécies de fauna e flora nativas. Sendo assim, o objetivo deste estudo foi avaliar a estrutura da paisagem de área urbanizada no bioma Mata Atlântica, utilizando técnicas de geoprocessamento e fundamentos de Ecologia de Paisagem, visando a fornecer subsídios à conservação florestal na paisagem urbana. Um mapa de uso e cobertura foi utilizado para quantificar a cobertura florestal e para avaliar a estrutura da paisagem. As métricas de paisagem quantificaram área (AREA), forma (SHAPE) e proximidade (NEAR) dos remanescentes florestais, fornecendo indicadores da estrutura da paisagem. A cobertura florestal representa $22,9 \%$ da área de estudo, sendo apenas 3,6\% dos fragmentos maiores que 50 ha. A grande maioria dos fragmentos (83\%) tem área menor que 10 ha. Apenas três remanescentes são maiores que 500 ha, sendo que o maior deles localiza-se na Floresta Nacional de Ipanema. A maioria dos fragmentos florestais (80\%) localiza-se em propriedades particulares. A paisagem foi diagnosticada como altamente antropizada e fragmentada, porém com remanescentes próximos entre si (NEAR $<50 \mathrm{~m}$ ), potencializando a movimentação das espécies na matriz antrópica. Cenários de exclusão dos pequenos fragmentos demonstraram a fragilidade da matriz sem o apoio deles, pois os pequenos remanescentes contribuem para a conexão entre os maiores. O campo antrópico de vegetação pioneira é a segunda classe de uso de maior cobertura na área de estudo (20,4\%), atrás somente da área urbanizada (25\%). Ações para a manutenção da biodiversidade em paisagens antropizadas, como as da área de estudo, devem priorizar projetos de restauração de campos antrópicos, incentivos à conservação de remanescentes em propriedades particulares e a manutenção de pequenos fragmentos na paisagem, a fim de promover a conexão entre as grandes áreas florestais. Os planejamentos urbano e ambiental das cidades devem ser alinhados e baseados em estudos científicos sobre a espacialização da vegetação no ambiente urbano.
\end{abstract}

Palavras-chave: Métricas da paisagem; Conservação florestal; Floresta urbana

Engenheira Civil, Pós-graduanda do Programa de Pós-Graduação em Planejamento e Uso de Recursos Renováveis (PPGPUR), Universidade Federal de São Carlos (UFSCar), Rod. João Leme dos Santos, km 110, CEP 18052-780, Sorocaba (SP), Brasil. marinapannunzio@gmail.com (ORCID: 0000-0002-9343-331X)

II Bióloga, Dra., Pesquisadora no Departamento de Ecologia, Instituto de Biociências, Universidade de São Paulo, Rua do Matão, 321, CEP 05508-900, São Paulo (SP), Brasil. kaline.mello@usp.br (ORCID: 0000-0001-7873-3042)

III Engenheira Florestal, Dra., Professora Associada no Departamento de Ciências Ambientais, Universidade Federal de São Carlos, Rod. João Leme dos Santos, km 110, CEP 18052-780, Sorocaba (SP), Brasil. roavalen@ufscar.br (ORCID: 0000-0001-7273-7042) 


\begin{abstract}
Considering that urban sprawl and the increase in anthropogenic activities lead to natural habitats loss and fragmentation, threatening biodiversity, the conservation of urban forest remnants is extremely important for the persistence of native fauna and flora species. The objective of this study was to evaluate the landscape structure of an urbanized area in the Atlantic Forest biome, using geoprocessing techniques and fundamentals of Landscape Ecology, in order to provide subsidies for forest conservation in the urban landscape. A land use/land cover map was used to quantify forest cover and to evaluate the landscape structure. Landscape metrics quantified the area, shape and proximity of the forest remnants, providing indicators of the landscape structure. Forest covers $22.9 \%$ of the study area, with only $3.6 \%$ of the fragments larger than 50 ha. The majority of the fragments (83\%) has an area of less than 10 ha. Only three remnants are larger than 500 ha, the largest of them is located in Ipanema National Forest. Most forest fragments $(80 \%)$ are located in particular properties. The landscape was diagnosed as highly anthropized and fragmented, but with remnants close to each other (NEAR $<50 \mathrm{~m})$ potentiating the movement of species in the anthropic matrix. Scenarios of exclusion of small fragments demonstrated the fragility of the matrix without their support, since the small remnants contribute to the connection between the larger ones. The anthropic grassland is the second largest land use class in the study area (20.4\%), behind only the urbanized area (25\%). Actions to maintain biodiversity in anthropized landscapes such as the study area should prioritize the restoration of anthropic grasslands, incentives for forest conservation in private properties and the maintenance of small fragments in the landscape, in order to promote the connection between large forest areas. Urban and environmental planning of cities should be aligned and based on scientific studies on the spatialization of the vegetation in urban areas.
\end{abstract}

Keywords: Landscape metrics; Forest conservation; Urban forest

\title{
Introdução
}

O processo de urbanização e a intensificação das atividades humanas transformam as paisagens naturais em mosaicos antrópicos, ou seja, áreas de vegetação nativa são convertidas em pequenas manchas de hábitat, dispersas em uma matriz antrópica (VALENTE; PETEAN; VETTORAZZI, 2017). A fragmentação dos hábitats, por sua vez, impacta os processos ecológicos e, consequentemente, leva à perda de biodiversidade (TANNIER et al., 2016). Essas alterações na paisagem vêm se tornando cada vez mais preocupantes, visto que $55 \%$ da população mundial vivem atualmente em áreas urbanas (UN, 2018). Enfrentamos, assim, o desafio de planejar as paisagens urbanas no sentido de suprir as necessidades humanas e, ao mesmo tempo, garantir a proteção da biodiversidade e os serviços ecossistêmicos providos pelas florestas urbanas (SMITH et al., 2018).

As maiores áreas urbanas no Brasil estão localizadas no bioma Mata Atlântica, considerado um hotspot mundial, com $28 \%$ de remanescentes de sua cobertura original (REZENDE et al., 2018), a qual está altamente fragmentada (RIBEIRO et al., 2009). No Estado de São Paulo, com exceção dos grandes maciços na região serrana, os remanescentes de vegetação natural encontramse muitas vezes isolados em uma matriz agrícola ou urbana (RODRIGUES; BONONI, 2008). Dentre as formações vegetais existentes, a Floresta Estacional e as fisionomias de Cerrado, predominantes no interior do Estado, foram as mais devastadas em função de estarem localizadas em topografias que favorecem a prática de atividades agropecuárias (PEREIRA; VENTUROLI; CARVALHO, 2011). Com o crescimento agrícola no Estado, também houve a expansão das áreas urbanas, ameaçando ainda mais essas formações de vegetação nativa (BRANCALION et al., 2016). $\mathrm{O}$ alto grau de fragmentação desse bioma ameaça a persistência das espécies (BANKS-LEITE et al., 2014), sendo necessário o manejo das paisagens antrópicas para garantir a manutenção da biodiversidade.

Os remanescentes naturais de uma matriz urbana ou periurbana possuem papel fundamental para garantir a conservação da biodiversidade e o fornecimento de serviços ecossistêmicos essenciais para a população (ZHANG et al., 2019). Mesmo apresentando tamanhos pequenos ou representando corredores ripários na paisagem urbana, esses remanescentes possuem potencial para garantir o hábitat natural para a persistência de espécies (BARBOSA et 
al., 2017; VALENTE; PETEAN; VETTORAZZI, 2017). Os pequenos fragmentos cumprem o papel de redutores de isolamento nas paisagens fragmentadas, servindo como trampolins ecológicos (BOSCOLO et al., 2008), enquanto os corredores ripários conectam manchas de hábitats, sendo substancialmente utilizados por pássaros endêmicos do bioma Mata Atlântica (SEKERCIOGLU, 2009).

Porém, para garantir a permanência de espécies, esses fragmentos presentes no ambiente urbano precisam ter a estrutura necessária para abrigar as populações, permitindo, por exemplo, a conectividade com outros fragmentos da paisagem (IGNATIEVA; STEWART; MEURK, 2011). Assim, é necessária a identificação das áreas prioritárias para conservação e/ou restauração que atendam a esses critérios ecológicos.

A identificação das áreas prioritárias à manutenção da biodiversidade local e que possam manter ou restabelecer as funções ecológicas da paisagem e os serviços essenciais é o arcabouço para o sucesso de ações de conservação e restauração em áreas urbanas e periurbanas (GUZMÁN WOLFHARD; RAEDIG, 2019). O êxito nessa identificação depende do entendimento da distribuição e das características físicas dos fragmentos florestais, ou seja, do entendimento da estrutura da paisagem (SILVA; SOUZA, 2014). Utilizando os conceitos da Ecologia de Paisagem, a estrutura de uma paisagem pode ser interpretada por meio de avaliações dos padrões de uso e cobertura do solo, obtidos por meio de imagens de satélites, técnicas de geoprocessamento e métricas de paisagem (MCGARIGAL, 2015).

Assim, a compreensão da estrutura da paisagem em áreas urbanas é necessária para dar suporte ao seu planejamento, subsidiando técnica e cientificamente a implementação de políticas públicas e projetos de conservação e restauração florestal na busca por cidades mais sustentáveis, que permitam a conservação da biodiversidade e dos serviços ecossistêmicos (MELLO; TOPPA; CARDOSO-LEITE, 2016; GONTIJO et al., 2019). Nesse sentido, o objetivo principal desta pesquisa foi avaliar a estrutura da paisagem de área urbanizada no bioma Mata Atlântica, utilizando técnicas de geoprocessamento e fundamentos de Ecologia de Paisagens e visando a fornecer subsídios à conservação florestal na paisagem urbana.

\section{Material e métodos}

A área de estudo é constituída pelo município de Sorocaba, localizado no Estado de São Paulo, e por uma faixa em seu entorno, que possui aproximadamente $5 \mathrm{~km}$ de largura. A extensão da área de estudo totaliza, assim, 109.560 ha e representa a porção mais populosa da região metropolitana de Sorocaba (Figura 1).

Localizada na porção sul da Mata Atlântica brasileira, a área de estudo é constituída por uma paisagem urbanizada e altamente fragmentada, com remanescentes florestais pequenos e, muitas vezes, pouco conectados (MELLO; TOPPA; CARDOSO-LEITE, 2016). Os autores afirmam que, nessa região, apesar de ser de domínio do bioma Mata Atlântica, com predomínio de Floresta Estacional Semidecidual, existem remanescentes de Cerrado.

A área está situada na Bacia Hidrográfica do Rio Sorocaba e Médio Tietê, sendo o Rio Sorocaba um dos principais do Estado de São Paulo (SISTEMA INTEGRADO DE GERENCIAMENTO DE RECURSOS HÍDRICOS DO ESTADO DE SÃO PAULO, 2018). O Rio Pirajibu é o principal afluente do Rio Sorocaba (SISTEMA INTEGRADO DE GERENCIAMENTO DE RECURSOS HÍDRICOS DO ESTADO DE SÃO PAULO, 2018), que possui sua nascente em uma área particular na divisa dos municípios de Votorantim e Mairinque.

A área de estudo possui duas Unidades de Conservação (UC) de uso sustentável: a Floresta Nacional (FLONA) de Ipanema, que conta com área total de 5.069,73 ha (INSTITUTO CHICO MENDES DE CONSERVAÇÃO DA BIODIVERSIDADE, 2010) e está situada a oeste, e a Área de Proteção Ambiental (APA) de Itupararanga, situada a sudeste (SÃO PAULO, 2003). A APA Itupararanga foi criada para proteger o reservatório e tem área total de 93.356,75 ha. No entanto, somente 4.170 ha estão presentes na área de estudo. 


\section{Figura 1 - Localização de Sorocaba e entorno, Estado de São Paulo, Brasil}

Figure 1 - Location of Sorocaba and its surroundings, state of São Paulo, Brazil

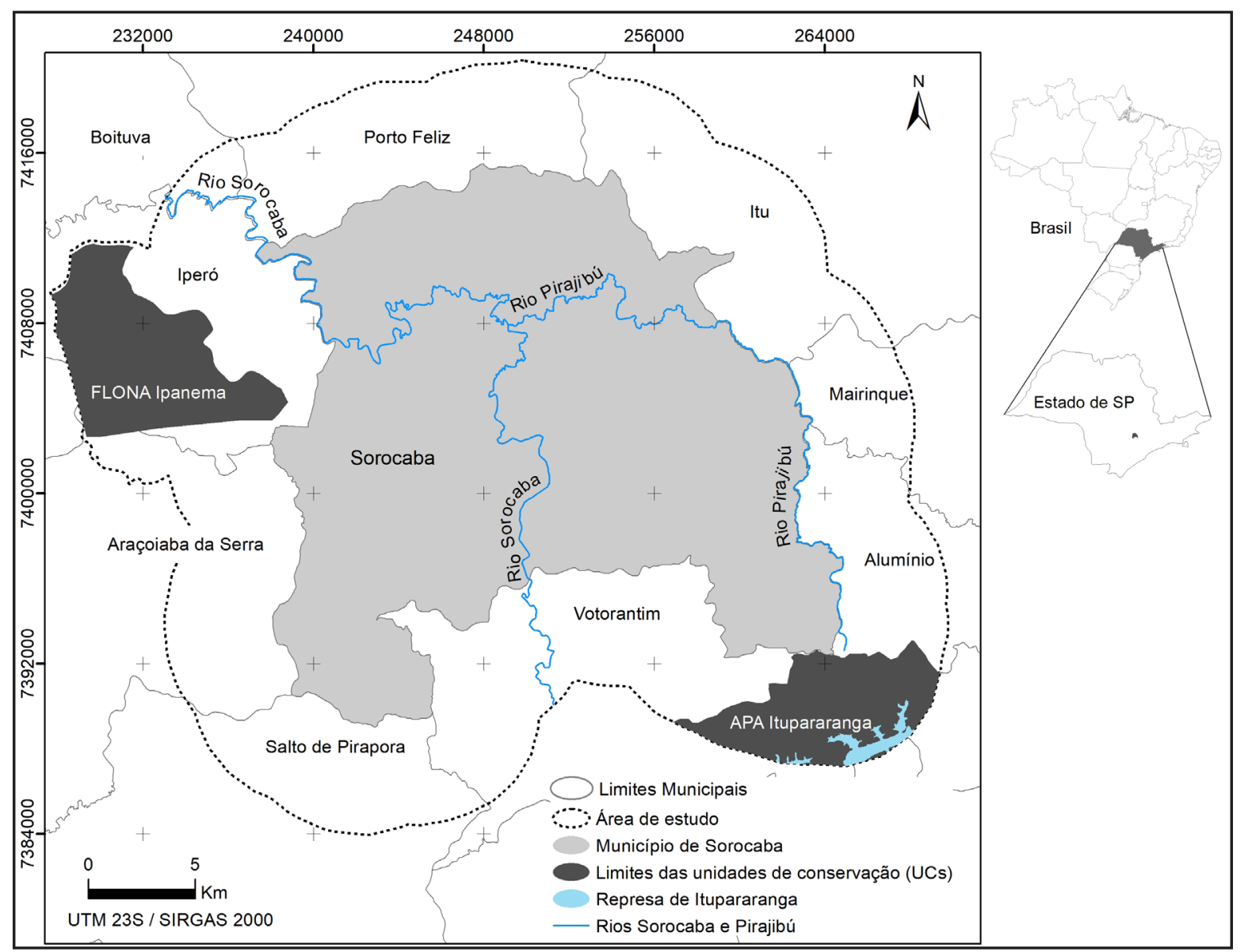

Fonte: Autoras (2019)

Para o mapeamento do uso e cobertura do solo foi utilizada uma imagem orbital obtida pelo sensor PAN10m, a bordo do satélite CBERS-4 (passagem de 28 de agosto de 2016), projeção Universal Transverse Mercator (UTM) e Datum Sirgas 2000. As bandas espectrais utilizadas foram as referentes aos comprimentos de onda do verde, vermelho e infravermelho, com os espectros do verde (B2) entre 0,52-0,59 $\mu \mathrm{m}$, do vermelho (B3) entre 0,63-0,69 $\mu \mathrm{m}$ e do infravermelho (B4) entre $0,77-0,89 \mu \mathrm{m}$, as quais permitiram elaborar uma composição colorida $(4 \mathrm{R} / 3 \mathrm{G} / 2 \mathrm{~B})$, destacando a vegetação na cor vermelha. A banda espectral infravermelha foi escolhida por sua capacidade de relacionar as características fisiológicas das folhas (conteúdo de água na estrutura celular superficial) com a elevada reflectância na banda, indicando que quanto mais alto valor espectral, maior a quantidade de biomassa presente. Segundo Meneses e Almeida (2012), o uso de bandas espectrais no comprimento de onda visível combinada com a banda do infravermelho aumenta o poder da detecção e identificação de objetos.

A rede hidrográfica utilizada neste estudo foi obtida no site da Companhia Ambiental do Estado de São Paulo (Cetesb -http://cetesb.sp.gov.br), sendo oriunda do projeto desenvolvido pelo Departamento de Águas e Energia Elétrica (DAEE), no sistema de referência Datum SAD 1969. Já a malha viária foi obtida no site do Departamento Nacional de Infraestrutura de Transportes (DNIT - http://dnit.gov.br), Datum Sirgas 2000, ambos na escala 1:50.000, projeção UTM e ambas disponíveis gratuitamente. Todos os arquivos vetoriais foram posteriormente convertidos para o Datum Sirgas 2000, projeção UTM e padronizados para a execução das métricas de paisagem. 


\section{Mapa de uso e cobertura do solo}

O mapa de uso e cobertura do solo foi produzido pelo método de classificação supervisionada multivariada de máxima verossimilhança (MAXVER) e finalizado no Sistema de Informação Geográfica (SIG).

No pré-processamento das imagens, realizaram-se as correções atmosférica e geométrica da imagem. Conforme descrito por Meneses e Almeida (2012), foram avaliados os histogramas das cenas e identificados os pixels que deveriam apresentar valores de reflectância nula. Esses pixels foram reclassificados para apresentar valores digitais iguais a zero, consequentemente corrigindo os demais. Esse procedimento é denominado "princípio da reflectância zero".

$\mathrm{Na}$ correção geométrica, as coordenadas reais foram padronizadas para o sistema de coordenadas UTM (Universal Transversa de Mercator) e sistema de referência Datum Sirgas 2000.

A definição das classes de uso e cobertura do solo foi estabelecida como: 1) Área florestal, identificada como Floresta Estacional Semidecidual do Bioma Mata Atlântica e formações florestais de Cerrado; 2) Silvicultura, sendo áreas ocupadas com plantios de Eucalyptus sp. ou Pinus sp.; 3) Culturas agrícolas temporárias, cultivo de plantas de curta ou média duração, com ciclo vegetativo inferior a um ano, como milho, alface, pimentão, mandioca, entre outras; 4) Campos antrópicos de vegetação pioneira, correspondem a campos originados de ações antrópicas com predomínio de plantas de pequeno porte; 5) Culturas agrícolas permanentes, áreas ocupadas com plantios de culturas perenes, como frutíferas e café; 6) Várzea / Zonas ripárias, vegetação rasteira em áreas úmidas e próximas a corpos d'água; 7) Áreas urbanizadas, áreas ocupadas com construções, os bairros rurais, os condomínios mais afastados dos principais centros urbanos, solos desnudos próximos a áreas urbanas e outros tipos de ocupação do solo onde existe a predominância de construções; 8) Áreas de mineração, áreas exploradas pela extração de minerais metálicos e minerais não metálicos; 9) Corpos d'água, formado por lagos e reservatório; 10) Cursos d'água, sendo os rios e córregos; e a 11) Malha viária, sendo as rodovias estaduais e federais pavimentadas.

As amostras de treinamento foram tomadas por digitalização de polígonos em tela, sobre as áreas que representavam as classes de uso e cobertura do solo previamente determinadas, para assim adquirir as estatísticas a serem empregadas pelo algoritmo de classificação (MENESES; ALMEIDA, 2012).

Após a tomada das amostras de treinamento, foi empregado o algoritmo MAXVER, cuja conceituação matemática foi estabelecida por Schalkoff(1992). De acordo com Meneses e Almeida (2012), o algoritmo de classificação associa os pixels da imagem a um número pré-determinado de classes individuais (amostras de treinamento), que representam os objetos do mundo real, sendo o resultado da classificação o reconhecimento dos padrões de uso de cobertura do solo.

$\mathrm{Na}$ busca por maior eficiência na classificação, foi aplicado um limiar, que é um valor real positivo (entre 0 e 1), empregado para o controle do nível de probabilidade no qual cada pixel pode ser considerado como pertencente ou não a uma classe (MENESES; ALMEIDA, 2012). Em nosso caso, foi adotado o valor de 0,05 para o limiar. Segundo Meneses e Almeida (2012), quanto menor o valor do limiar, mais rigorosa será a classificação, sendo que, desse modo, os pixels com menor probabilidade que o limiar estabelecido não são classificados.

Procurando atenuar o efeito da presença de pixels isolados, comuns em produtos de classificações com o algoritmo MAXVER, foi aplicado um filtro de mediana de 7 x 7 pixels como tratamento pós-classificação. O filtro de mediana é classificado como um filtro passa-baixa, em que o pixel central da máscara é substituído pela mediana dos seus vizinhos. Trata-se de um filtro de implementação complexa, devido à necessidade de um ordenamento dos valores, e foi utilizado nesta análise por ser um filtro de suavização que melhor preserva as bordas em uma imagem (MENESES; ALMEIDA, 2012).

Após sua aplicação, o produto final da classificação foi transformado em vetores e finalizado manualmente no SIG ArcGIS, tendo por base a própria composição colorida da 
imagem de satélite e imagens de alta resolução disponíveis do Google Earth (http://earth.google. com). A escala de trabalho do mapeamento manual foi de 1:25.000.

Para a verificação da exatidão do mapa final de uso e cobertura do solo, gerou-se uma malha de pontos (n amostral de 133 pontos), distribuídos de forma estratificada ao acaso, bem como o número de amostras (n), ambos estabelecidos por Eastman (2003) por meio das equações 1 e 2 .

$$
\begin{aligned}
& N=Z^{2} \times p \times q / e^{2} \\
& A=N / a
\end{aligned}
$$

Sendo: N: número de amostras; Z: valor padrão para um nível de confiança específico (no caso 95\%); p: percentual de exatidão esperado; q: 100 - p; A: número de amostras para a área de estudo; a: proporção da área de estudo (no caso 76,5\%).

Os 133 pontos amostrais foram verificados, tendo como referência as informações de campo, a própria imagem de satélite e imagens de alta resolução disponíveis do Google Earth (http://earth.google.com). No campo foram visitados 30 pontos, dos quais foram registradas as coordenadas geográficas, por meio de um receptor Global Positioning System (GPS) de navegação e alguns deles fotografados.

Estimou-se a exatidão do mapa de uso de cobertura usando uma matriz de confusão, onde se consideraram os valores de $85 \%$ para o percentual de exatidão esperado e $5 \%$ para o erro admissível (EASTMAN, 2003) e o índice Kappa (LANDIS; KOCH, 1977). O índice Kappa indica a qualidade da classificação em uma escala de 0 a 1, onde o valor de 1 é considerado perfeito (LANDIS; KOCH, 1977).

\section{Estrutura da paisagem e dos fragmentos florestais}

A classe área florestal foi extraída do mapa de uso e cobertura do solo e caracterizada por meio das métricas tradicionais de Ecologia de Paisagem, desenvolvidas por McGarigal (2015) em ambiente SIG ArcGis. A categorização aconteceu: a) em nível de classe, por meio das métricas: índices de tamanho dos fragmentos, índices de forma e índices de proximidade, incluindo os respectivos valores de desvio padrão e coeficientes de variação; e b) em nível de fragmentos, por meio das métricas: índice de área (AREA), índice de forma (SHAPE) e índice de proximidade (NEAR) (MCGARIGAL, 2015), conforme descrito na Tabela 1.

Para um melhor entendimento da estrutura dos fragmentos florestais, usando os três índices em nível de fragmento (AREA, SHAPE, NEAR), os remanescentes foram agrupados em classes elaboradas, onde foram calculados o número total e a percentagem de fragmento em relação ao número total de remanescentes florestais e, também a área total e a porcentagem de área em relação à área total de remanescentes florestais. forma:

O agrupamento dos remanescentes para cada índice de fragmento foi feito da seguinte

i. Índices de área (AREA): a partir do cálculo da área de todos os remanescentes florestais, os fragmentos foram agrupados em cinco classes de tamanho (em hectare), sendo elas, menores que 1 ; entre 1 e 10 ; entre 10 e 50 ; entre 50 e 500 ; acima de 500 .

ii. Índices de forma (SHAPE): a partir do cálculo do índice de forma de todos os remanescentes florestais, que indica a complexidade da forma dos fragmentos quando comparada a uma forma padrão ideal, próxima de círculo, os fragmentos foram agrupados em cinco classes SHAPE (sem unidade), sendo elas, entre 1 e 2 ; entre 2 e 3 ; entre 3 e 4 ; entre 4 e 5 e acima de 5.

iii. Índices de proximidade (NEAR): a partir do cálculo do índice de proximidade de 
todos os remanescentes florestais, que quantifica a distância euclidiana, ou borda-a-borda, os fragmentos foram agrupados em cinco classes NEAR (metros), sendo elas, menor que 50; entre 50 e 100; entre 100 e 200; entre 200 e 500; acima de 500.

\section{Tabela 1 - Métricas de paisagem em níveis de fragmento e de classe de uso e cobertura do solo utilizadas para caracterizar a estrutura da paisagem da área de estudo}

Table1 - Landscape metrics in fragment levels, land use/land cover class employed to the landscape structure characterization of the study area

\begin{tabular}{|c|c|c|}
\hline \multirow{9}{*}{$\begin{array}{l}\text { Índices de } \\
\text { classe }\end{array}$} & \multirow{4}{*}{ Índices de tamanho } & a) Número de fragmentos \\
\hline & & b) Tamanho médio dos fragmentos \\
\hline & & c) Desvio padrão do tamanho médio de fragmentos \\
\hline & & d) Coeficiente de variação do tamanho médio de fragmentos \\
\hline & \multirow{2}{*}{ Índices de forma } & e) Índice de forma média \\
\hline & & f) Índice de forma média ponderado pela área \\
\hline & \multirow{3}{*}{ Índices de proximidade } & g) Distância média entre fragmentos \\
\hline & & h) Desvio padrão para a distância média entre fragmentos \\
\hline & & i) Coeficiente de variação para a distância média entre fragmentos \\
\hline \multirow{3}{*}{$\begin{array}{l}\text { Índices de } \\
\text { fragmentos }\end{array}$} & Índice de área & k) Área do fragmento (AREA) \\
\hline & Índice de forma & 1) Índice de forma (SHAPE) \\
\hline & Índice de proximidade & m) Distância média entre fragmentos (NEAR) \\
\hline
\end{tabular}

Fonte: Adaptada de McGarigal (2015)

Com a finalidade de compreender a importância dos pequenos fragmentos nas estimativas de isolamento dos remanescentes florestais da paisagem, cenários de exclusão dos remanescentes menores foram criados a partir da subtração dos fragmentos pertencentes às seguintes classes de tamanho (ha), realizadas em ambiente SIG.: $<1 ;<2 ;<5 ;<10 ;<20$; e $<50$.

\section{Resultados e discussão}

O mapa de uso e cobertura do solo da área de estudo (Figura 2) apresentou uma exatidão global de 93,23\%, tendo a classe Área florestal exatidão de $88,46 \%$, percentual considerado aceitável (sendo 85\% o mínimo admissível) (EASTMAN, 2003). Quanto ao índice Kappa, obtevese para o mapa o valor de 0,93 , classificação considerada excelente, de acordo com Landis e Koch (1977).

A área do município de Sorocaba e seu entorno é composta por diferentes classes de uso e cobertura do solo, com os remanescentes florestais representando $22,9 \%$ da paisagem. Tal valor é semelhantemente ao já observado em Sorocaba por Mello, Toppa e Cardoso-Leite (2016), que registraram cerca de $17 \%$ da cobertura do solo identificada como área de floresta nativa para o município. Tanto o percentual de $22,9 \%$ quanto o de $17 \%$ são preocupantes para a conservação da biodiversidade, visto que paisagens com menos de $30 \%$ de cobertura de vegetação nativa apresentam comprometimento das funções ecológicas e, consequentemente, grande perda de biodiversidade (BANKS-LEITE et al., 2014). Rezende et al. (2018), em estudo recente, apontaram que, no bioma Mata Atlântica, os remanescentes da sua cobertura original representam $28 \%$, enquanto as áreas antrópicas ocupam $65 \%$. Isso evidencia o padrão de uso do solo, modificado 
por ações humanas nesse bioma, como também identificaram Ribeiro et al. (2009), Mello, Toppa e Cardoso-Leite (2016) e Valente, Petean e Vettorazzi (2017).

\section{Figura 2 - Uso e cobertura do solo de Sorocaba e entorno, Estado de São Paulo, Brasil}

Figure 2 - Land use/land cover of Sorocaba and its surroundings, state of São Paulo, Brazil

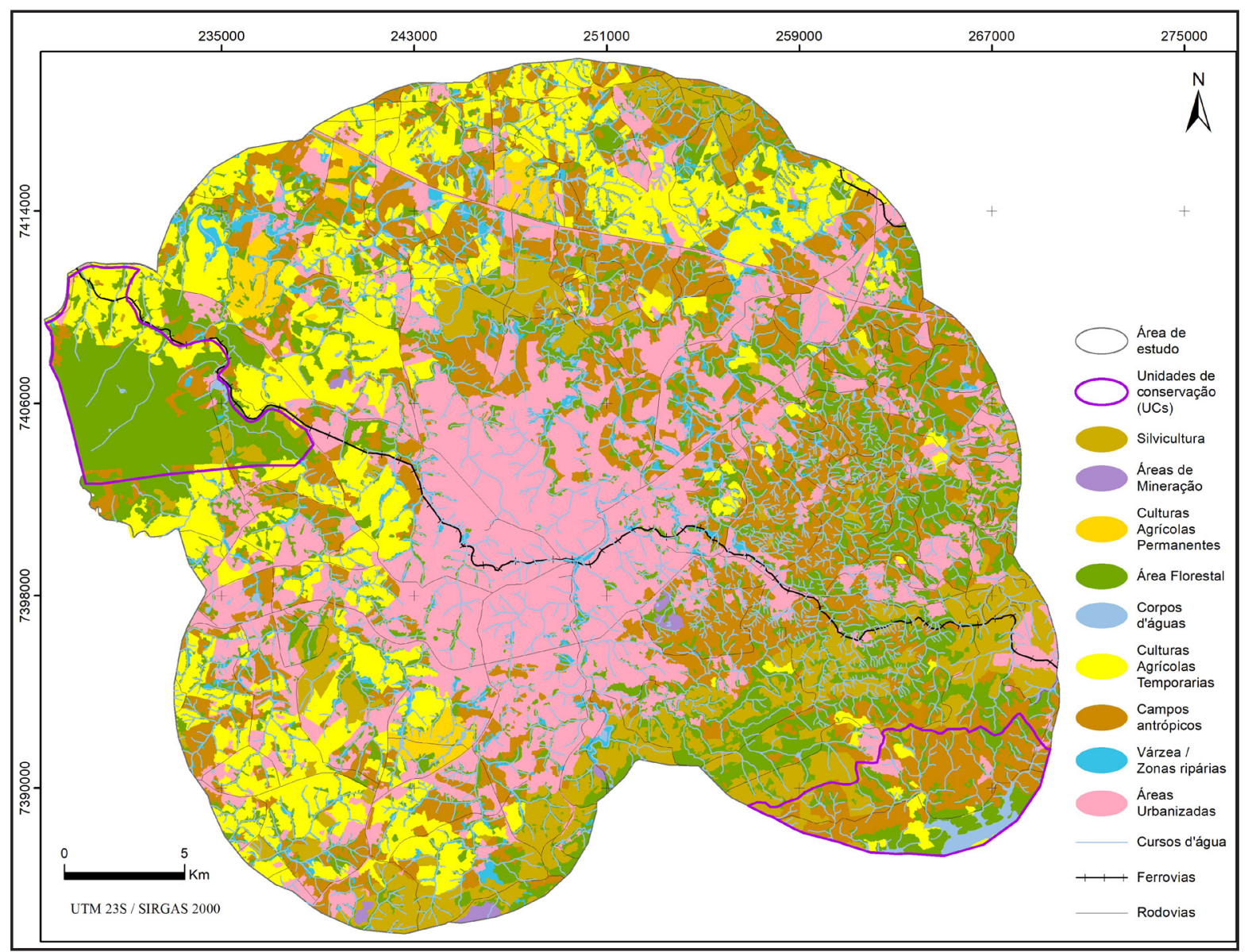

Fonte: Autoras (2019)

O uso do solo de maior representatividade na área de estudo é o urbano, incidindo sobre $25 \%$ de sua área total. Como outros usos, têm-se os campos antrópicos de vegetação pioneira $(20,4 \%)$, as culturas agrícolas temporárias $(17 \%)$, as culturas agrícolas permanentes $(1,4 \%)$, a silvicultura $(9 \%)$ e, ainda, pequenas áreas ocupadas por mineração $(0,3 \%)$. Juntos eles correspondem a cerca de $73 \%$ da área de estudo, a qual também possui lagos, rios, córregos e reservatórios, representando $1,2 \%$ de sua área total, além de zonas de várzea, que equivalem a $2,9 \%$. Dessa forma, a área de estudo, localizada no interior do Estado de São Paulo, apresenta-se como uma paisagem altamente antropizada, sendo a área urbanizada a classe de maior representatividade.

Com relação à estrutura da cobertura florestal, obteve-se um total de 1.854 remanescentes florestais, os quais estão distribuídos pelo município de Sorocaba e seu entorno. Esses remanescentes possuem área variando entre 0,10 ha e 4.600 ha, com tamanho médio de 13,52 ha (desvio padrão $=132,27$ ha; coeficiente de variação $=978,29 \%$ ), conforme pode ser observado na Tabela 2. 
Tabela 2 - Número de fragmentos, proporção de fragmentos florestais (\%), área total (ha), área em porcentagem (\%), desvio padrão e coeficientes de variação (\%) por classe de tamanho (ha) da floresta nativa para Sorocaba e entorno, Estado de São Paulo, Brasil

Table 2 - Number of forest fragments, proportion of forest fragments (\%), total area (ha), area in percentage (\%), standard deviation and coefficient of variation (\%) for size class (ha) of native forest for Sorocaba and its surroundings, state of São Paulo, Brazil

\begin{tabular}{lcccccc}
\hline $\begin{array}{c}\text { Classes de } \\
\text { distribuição / } \\
\text { área (ha) }\end{array}$ & $\begin{array}{c}\text { Número de } \\
\text { fragmentos }\end{array}$ & $\begin{array}{c}\text { Proporção de } \\
\text { fragmentos } \\
\text { florestais (\%) }\end{array}$ & $\begin{array}{c}\text { Área } \\
\text { total (ha) }\end{array}$ & $\begin{array}{c}\text { Área em } \\
\text { porcentagem } \\
\text { (\%) }\end{array}$ & $\begin{array}{c}\text { Desvio } \\
\text { padrão }\end{array}$ & $\begin{array}{c}\text { Coeficiente } \\
\text { de variação } \\
\text { (\%) }\end{array}$ \\
\hline$<\mathbf{1}$ & 542 & 29,23 & 264,91 & 1,06 & 0,29 & 59,54 \\
$\mathbf{1} \mathbf{- 1 0}$ & 993 & 53,56 & $3.419,60$ & 13,64 & 2,23 & 64,69 \\
$\mathbf{1 0}-\mathbf{5 0}$ & 253 & 13,65 & $5.389,42$ & 21,50 & 9,90 & 46,49 \\
$\mathbf{5 0}-\mathbf{5 0 0}$ & 63 & 3,40 & $7.213,91$ & 28,78 & 78,36 & 68,43 \\
$>\mathbf{5 0 0}$ & 3 & 0,16 & $8.778,62$ & 35,02 & $1.380,37$ & 47,17 \\
Total & 1.854 & 100,00 & $25.066,46$ & 100,00 & 132,27 & 978,29 \\
\hline
\end{tabular}

Fonte: Autoras (2019)

Em uma matriz antrópica em que predominam áreas urbanas e campos antrópicos, a maioria dos remanescentes florestais da paisagem (cerca de $83 \%$ do total) apresentaram AREA < 10 ha, representando aproximadamente $14 \%$ da área de floresta nativa, sendo que cerca de $29 \%$ têm AREA $<1$ ha. Os pequenos remanescentes florestais podem não ser suficientes para manter populações de espécies nativas em longo prazo, mas podem ser importantes para os animais migratórios ou funcionar como trampolins ecológicos (BARBOSA et al., 2017).

Apenas sessenta e seis fragmentos (3,56\%) apresentaram AREA $>50$ ha, sendo que três possuem AREA > 500 ha, evidenciando a predominância de pequenos fragmentos na paisagem. Os maiores fragmentos de vegetação natural concentram-se nas zonas periurbanas, onde $o$ parcelamento do solo é heterogêneo, sendo composto por pequenas propriedades rurais, além de loteamentos residenciais e industriais que se expandem nas zonas periféricas dos municípios (MELLO; TOPPA; CARDOSO-LEITE, 2016). Esses remanescentes maiores são de grande importância para a manutenção da biodiversidade e devem ser foco de projetos de conservação, pois, em função de apresentarem maior estabilidade contra variações ambientais e oferecem melhores perspectivas de sustentar espécies em longo prazo, eles desempenham papel de reservas naturais (RIBEIRO et al.,2009).

O maior fragmento encontrado na paisagem está na FLONA Ipanema, localizada na região oeste da área de estudo (Figura 3). Esse fragmento possui área aproximada de 4.600 ha, o que equivale a $18,35 \%$ da área total de floresta nativa da paisagem, representando o maior remanescente de Floresta Estacional Semidecidual da região. Assim, essa UC configura-se como um fragmento florestal chave para a manutenção da biodiversidade da paisagem, não só da área de estudo, como também de formações florestais do ecótono entre os biomas Mata Atlântica e Cerrado (INSTITUTO CHICO MENDES DE CONSERVAÇÃO DA BIODIVERSIDADE, 2010).

Os remanescentes localizados na APA Itupararanga têm apenas $0,94 \%$ de representação florestal no recorte de área estudado, totalizando, juntamente com a FLONA Ipanema, aproximadamente $20 \%$ de cobertura florestal dentro de UCs. É importante ressaltar que este estudo não contempla toda a extensão dessa UC. Entretanto, na porção estudada, foi identificada a predominância de usos do solo como campos antrópicos e áreas agrícolas, o que ressalta a importância de projetos de restauração florestal, visto que, além da conservação da biodiversidade, 
essa UC é responsável pela manutenção do principal manancial da região (SÃO PAULO, 2003).

\section{Figura 3 - Distribuição dos remanescentes de floresta nativa, considerando classes de tamanho para Sorocaba e entorno, Estado de São Paulo, Brasil}

Figure 3 - Distribution of native forest remnants considering size classes in Sorocaba and its surroundings, state of São Paulo, Brazil

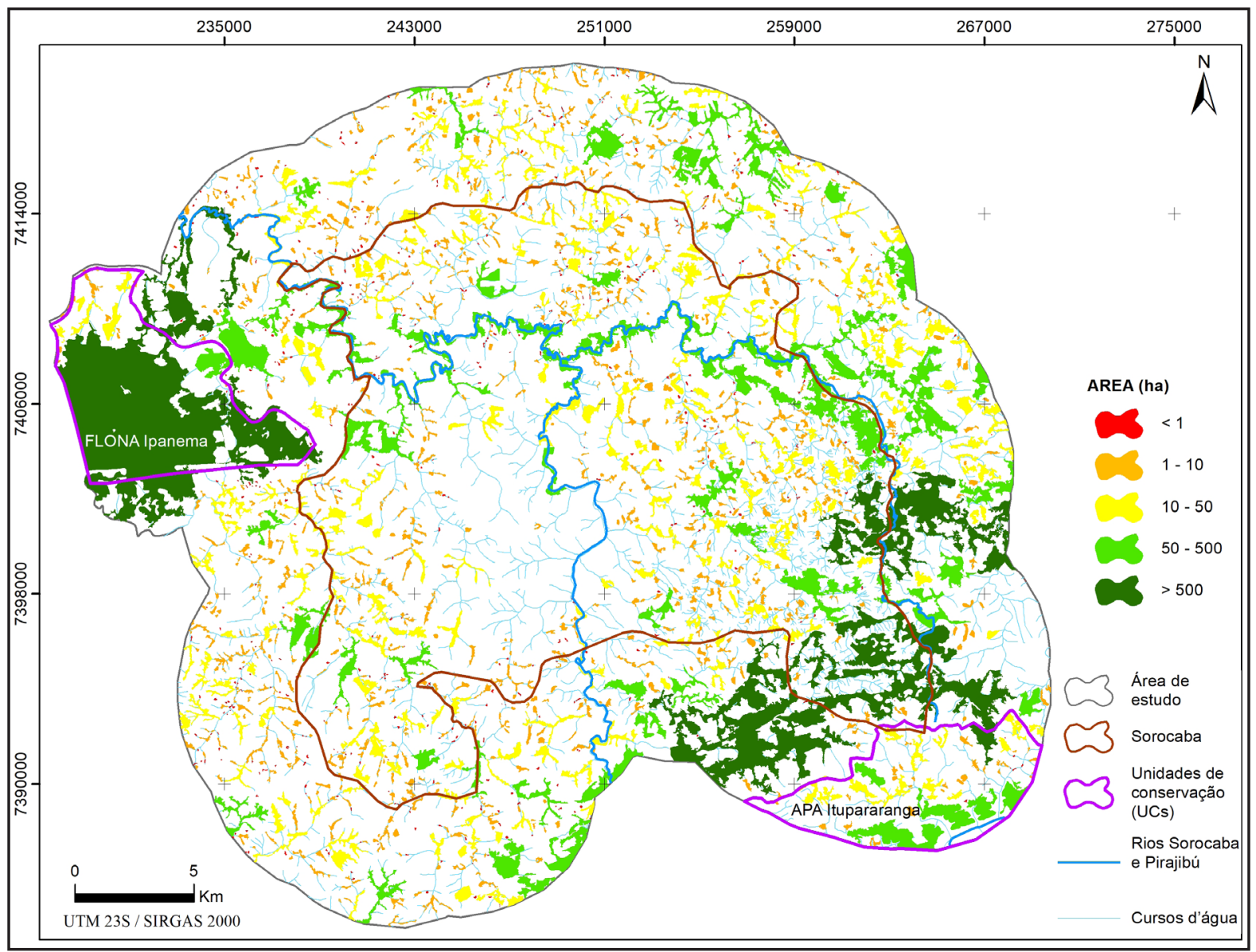

Fonte: Autoras (2019)

Os outros dois fragmentos maiores da paisagem (AREA > 500 ha) estão nas porções leste e sudeste do município, próximos ao limite dos municípios de Sorocaba, Alumínio e Mairinque, em propriedades particulares e associados aos cursos d'água. $O$ fragmento localizado na região leste da área de estudo tem cerca de 1.230 ha, e o outro, localizado a sudeste, possui aproximadamente 2.900 ha. Juntos, eles totalizam $16,48 \%$ da área remanescente florestal da paisagem. Na medida em que a grande maioria dos remanescentes florestais da área de estudo está localizada em propriedades particulares (aproximadamente 80\%), esses fragmentos são considerados elementos essenciais para a matriz urbana em questão. Fica, assim, patente a necessidade de incentivos para conservação da vegetação nativa em propriedades particulares, garantindo a manutenção das florestas urbanas como forma de preservar a biodiversidade e o provimento de serviços ecossistêmicos à população (MELLO; TOPPA; CARDOSO-LEITE, 2016).

Avaliando a proximidade entre os remanescentes, observa-se que a maior parte dos fragmentos da paisagem $(54 \%$ do total) possui pelo menos um remanescente com proximidade mínima de $50 \mathrm{~m}$ de distância de outro (Figura 4), o que potencializa a dispersão das espécies nativas pela paisagem (BOSCOLO et al., 2008). O valor médio de proximidade foi de $80,8 \mathrm{~m}$ (desvio 
padrão $=93,44 \mathrm{~m}$; coeficiente de variação $=119,4 \%$ ) entre fragmentos florestais. Para Martensen, Pimentel e Metzger (2008), em ações de gerenciamento de paisagens, a curta distância entre fragmentos é essencial para garantir a conservação da biodiversidade em ambientes antropizados.

A proximidade entre os fragmentos na área de estudo proporciona uma ligação, ou uma rede ecológica, entre as florestas situadas em propriedades particulares, localizadas nas zonas periurbanas do município, e as UCs existentes, o que é um fator que contraria diretamente a fragmentação florestal e contribui para a manutenção da biodiversidade nas áreas protegidas, conectando-as entre si (MELLO; TOPPA; CARDOSO-LEITE, 2016), além de servir à conservação dos serviços ecossistêmicos, essenciais à vida humana. Dessa forma, estudos geoespaciais que tragam informações quanto à distribuição espacial das florestas nativas devem amparar decisões do poder público quanto à expansão urbana territorial, modificações no zoneamento e supressão florestal, sendo peça fundamental nas decisões de planejamento e ordenamento territorial (GONTIJO et al., 2019).

\section{Figura 4 - Distribuição dos remanescentes de floresta nativa por classes de índice NEAR} para Sorocaba e entorno, Estado de São Paulo, Brasil

Figure 4 - Distribution of native forest remnants considering NEAR index in Sorocaba and its surroundings, state of São Paulo, Brazil

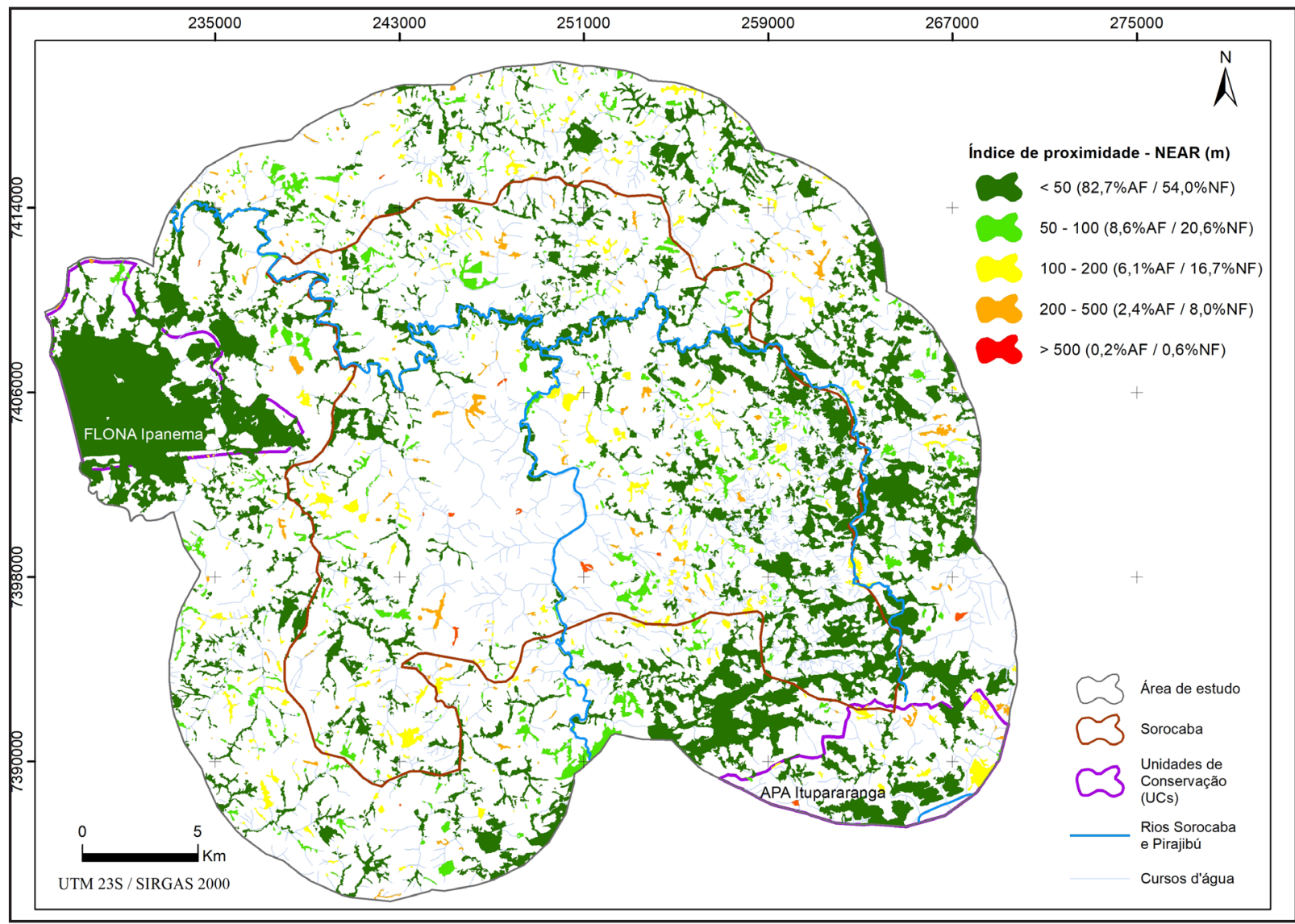

Fonte: Autoras (2019)

Em que: Porcentagem da área (\%AF) e do número de remanescentes de floresta nativa (\%NF).

A supressão florestal, por exemplo, se aplicada a todos os fragmentos com tamanho menor do que 50 ha, elevaria a distância média entre fragmentos a 567,7 m (Figura 5). Portanto, 
tratando-se de áreas altamente fragmentadas como a área de estudo, o papel dos pequenos remanescentes é essencial para a redução do isolamento dos fragmentos florestais na paisagem e para a manutenção da biodiversidade regional, servindo como trampolins ecológicos entre as grandes áreas florestais, sendo áreas protegidas ou não (RIBEIRO et al., 2009; REZENDE et al., 2018). Fragmentos naturais isolados inviabilizam a persistência de espécies, principalmente das endêmicas da Mata Atlântica. Como ressaltaram Boscolo et al. (2008) e Sekercioglu (2009), as espécies endêmicas muitas vezes não conseguem transpor distâncias na matriz aberta maiores de $50 \mathrm{~m}$.

Figura 5 - Distância média entre fragmentos (MNN) de remanescentes de floresta nativa para diferentes cenários de exclusão de fragmentos por classe tamanho (ha) para Sorocaba e entornos, Estado de São Paulo, Brasil

Figure 5 - Mean distance between fragments (MNN) of native forest remnants for different scenarios of fragment exclusion by size class (ha) for Sorocaba and its surroundings, state of São Paulo, Brazil

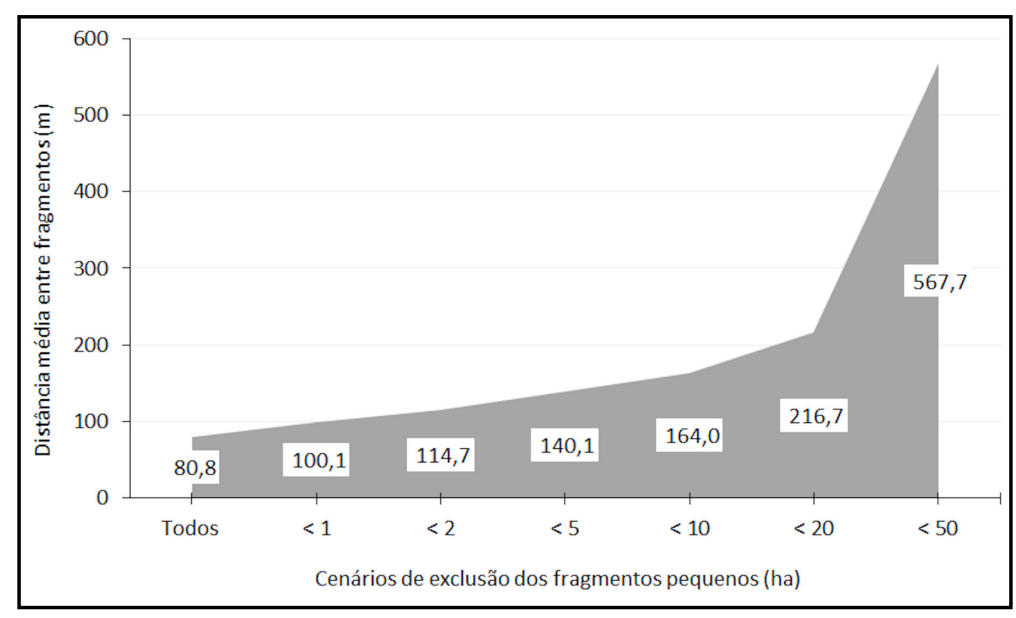

Fonte: Autoras (2019)

As regiões leste e sudeste da área de estudo, onde há uma grande concentração de nascentes e riachos, reúnem uma expressiva quantidade de remanescentes naturais que acompanham os cursos d'água, os quais formam o Rio Pirajibu e são importantes mananciais da região, apresentando índice $\mathrm{NEAR}<50 \mathrm{~m}$. Além disso, essa região é a mais declivosa da área de estudo, o que explica a presença de maior cobertura florestal, uma vez que seu relevo dificulta a expansão urbana e agrícola (MELLO; TOPPA; CARDOSO-LEITE, 2016).

Os contínuos de floresta formados por zonas ripárias também contribuem para a manutenção da vegetação natural em paisagens antropizadas. As zonas ripárias são áreas protegidas por lei (Lei n. 12.651, de 25 de maio de 2012, antigo Código Florestal) e, além de contribuírem para a conectividade da paisagem, sendo utilizadas como corredores ecológicos para a fauna, desempenham um papel significativo na manutenção da qualidade da água e na proteção do solo (BRANCALION et al., 2016; VALENTE; PETEAN; VETTORAZZI, 2017). Grande parte da vegetação nativa remanescente na área de estudo é composta por regiões periféricas ao centro urbano, em encostas de grande altitude e zonas ripárias, como já haviam diagnosticado Mello, Toppa e Cardoso-Leite (2016), destacando a importância da conservação dessas áreas nos ambientes urbanos e periurbanos. Algumas áreas adjacentes ao Rio Sorocaba não apresentaram cobertura florestal, o que evidencia a importância de ações de restauração da vegetação ripária desse importante rio do Estado de São Paulo. 
Por estarem associados a cursos d'água, o grupo dos maiores remanescentes (AREA > 500 ha) apresentou forma irregular e distante da considerada ideal, que é um círculo (MCGARIGAL, 2015). Sua forma irregular foi representada pelos maiores valores do índice SHAPE (SHAPE > 5). A maioria dos remanescentes da paisagem $(69,4 \%)$ tem valores de SHAPE entre 1 e 2 . Contudo, são pequenos fragmentos que, somados, representam apenas $12,8 \%$ da área florestal, ou 3.200 ha, aproximadamente.

Tambosi (2008) ressalta que a forma mais alongada dos fragmentos ripários está ligada ao acompanhamento dos cursos d'água, o que justifica os altos valores de índice de forma dos fragmentos, sendo que os maiores remanescentes se encontram junto aos cursos d'água e distantes do centro urbano, com relevante parcela a leste da área de estudo, acompanhando o Rio Pirajibu (Figura 6).

Considerando que o valor um (1) no índice SHAPE indica que o fragmento tem forma circular e que, quanto mais se distancia desse valor, mais complexa será sua forma (MCGARIGAL, 2015), pode-se dizer que os maiores remanescentes têm forma mais complexa do que os menores, com exceção da área de floresta nativa situada no interior da FLONA Ipanema. O índice de forma médio e o índice de forma médio ponderado pela área atingiram, respectivamente, os valores de 1,94 e 6,56 para a paisagem em estudo.

Figura 6 - Distribuição dos remanescentes de floresta nativa por classes de índice de forma para Sorocaba e entornos, Estado de São Paulo, Brasil

Figure 6 - Distribution of native forest remnants considering shape index in Sorocaba and its surroundings, state of São Paulo, Brazil

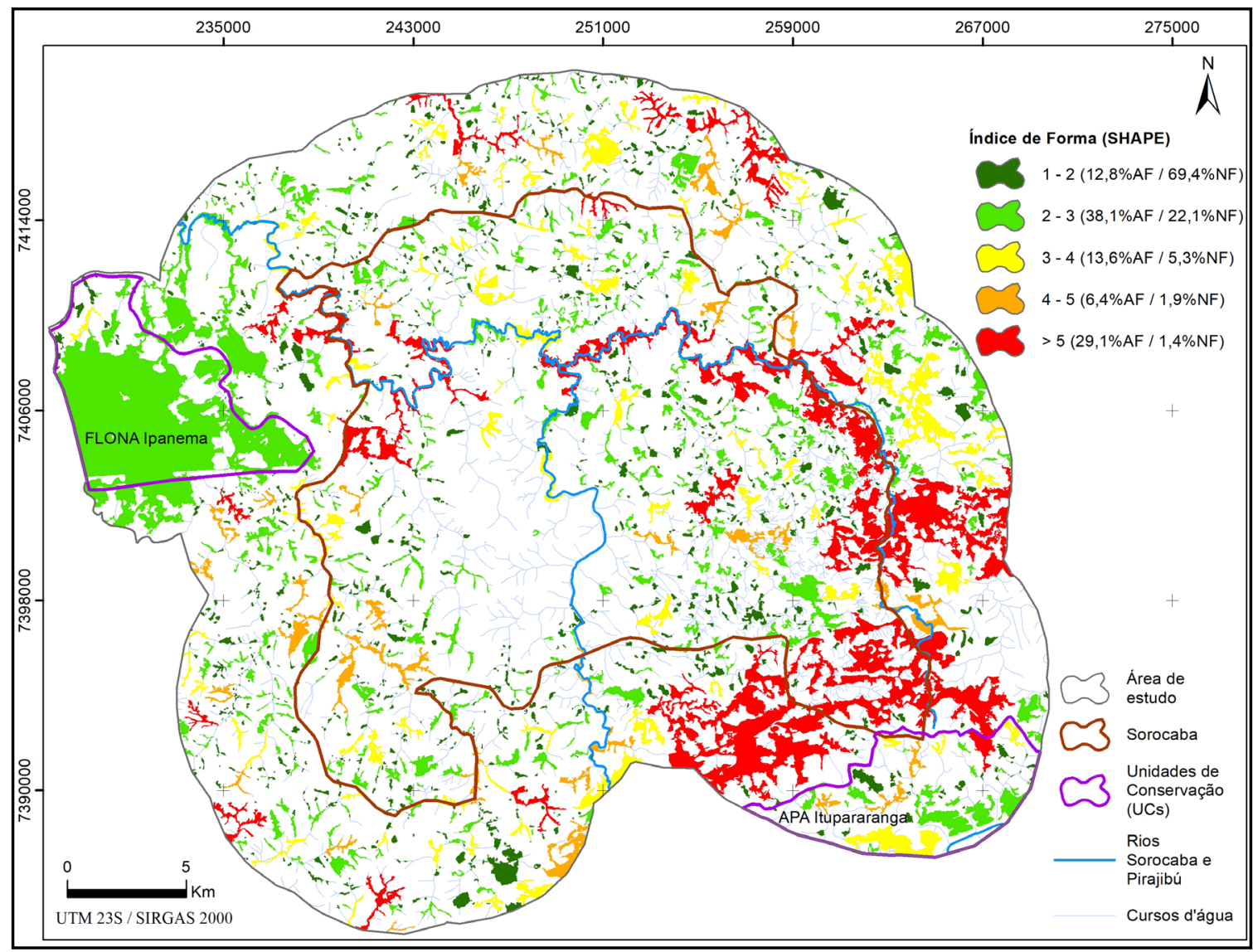

Fonte: Autoras (2019)

Em que: Porcentagem da área (\%AF) e do número de remanescentes de floresta nativa (\%NF). 
Algumas zonas ripárias, principalmente adjacentes ao Rio Sorocaba, que não apresentaram cobertura florestal foram caracterizadas como campos antrópicos de vegetação pioneira. Visto que os campos antrópicos, que representam $20,4 \%$ da paisagem, são áreas não exploradas produtivamente, eles podem ser considerados adequados para projetos de restauração florestal, principalmente em paisagens tropicais como a Mata Atlântica (LATAWIEC et al., 2015).

Estudos recentes, tal como o de Rezende et al. (2018), apontaram um crescimento das áreas em regeneração na Mata Atlântica, fenômeno relacionado à regeneração de vegetação nativa em campos degradados. Uma vez que as áreas urbanas são áreas consolidadas e dificilmente serão convertidas, os campos antrópicos presentes na paisagem urbana, como no caso da área de estudo, são áreas importantes para projetos de restauração florestal dos municípios.

\section{Conclusão}

A área de estudo possui uma paisagem antropizada com alta fragmentação florestal, uma vez que a grande maioria dos remanescentes apresenta área inferior a 10 ha. Contudo, a paisagem também abriga três grandes fragmentos, estando o maior deles localizado na FLONA Ipanema, que representa a área florestal mais importante da região, e os outros dois em propriedades particulares. A maioria dos remanescentes florestais da área de estudo (80\%) encontra-se em áreas particulares, o que ressalta a importância de incentivos à conservação de fragmentos nessas áreas como forma de manutenção da biodiversidade e dos serviços ecossistêmicos essenciais à população.

A alta proximidade entre os remanescentes florestais na paisagem de estudo mostra o potencial para movimentação de espécies na paisagem antrópica. Os cenários de exclusão de pequenos fragmentos possibilitaram identificar a necessidade de permanência dos remanescentes menores na paisagem urbana, pois eles contribuem para a conexão entre os remanescentes maiores.

Grande parte dos remanescentes florestais na paisagem está associada à rede hidrográfica, sendo compostos muitas vezes por corredores ripários e apresentando, em sua maioria, formas complexas devido aos contornos da hidrografia. Observou-se também que algumas áreas adjacentes ao Rio Sorocaba, um dos principais rios do Estado de São Paulo, não apresentam cobertura florestal ripária, sendo classificadas como campos antrópicos de vegetação pioneira. Essa classe representa atualmente $20,4 \%$ da paisagem e é considerada como potencial para o direcionamento de projetos de restauração florestal.

Assim, ações de conservação e restauração de vegetação ripária, incentivos à conservação de fragmentos de vegetação nativa em propriedades privadas, manutenção de pequenos fragmentos e implantação de projetos de restauração florestal em campos antrópicos, que aumentem a conexão entre áreas florestais maiores, são importantes para a manutenção da biodiversidade em paisagens urbanas. Estudo como este, que direciona esforços para a conservação da biodiversidade e utiliza como base dados espaciais, pode fornecer subsídios técnicos e científicos para projetos de planejamento urbano e ambiental, que devem ser alinhados para que toda a sociedade possa usufruir dos benefícios de um meio ambiente sustentável.

\section{Agradecimentos}

Agradecemos ao Programa de Pós-Graduação em Planejamento e Uso de Recursos Renováveis (PPGPUR) da Universidade Federal de São Carlos (UFSCar) por financiamento de valores relacionados a publicação do referente artigo. 


\section{Referências}

BANKS-LEITE, C. et al. Using ecological thresholds to evaluate the costs and benefits of setasides in a biodiversity hotspot. Science, Pensilvânia, v. 345, n. 6200, p. 1041-1045, aug. 2014.

BARBOSA, K. V. de C. et al. Use of small Atlantic Forest fragments by birds in Southeast Brazil. Perspectives in Ecology and Conservation, Amsterdam, v. 15, n. 1, p. 42-46, jan. 2017.

BOSCOLO, D. et al. Importance of Interhabitat Gaps and Stepping-Stones for Lesser Woodcreepers (Xiphorhynchus fuscus) in the Atlantic Forest, Brazil. Biotropica, New Jersey, v. 40, n. 3, p. 273-276, may 2008.

BRANCALION, P. H. S. et al. A critical analysis of the Native Vegetation Protection Law of Brazil (2012): updates and ongoing initiatives. Natureza \& Conservação, Curitiba, v. 14, p. 1-15, abr. 2016.

EASTMAN, J. R. IDRISI Kilimanjaro guide to GIS and image processing. Worcester, MA: Clark Labs, Clark University, 2003.

GONTIJO, G. A. B. et al. Análise do atendimento ao Código Florestal e a regularização ambiental por unidades de bacias hidrográficas. Ciência Florestal, Santa Maria, v. 29, n. 4, p. 1538, dez. 2019.

GUZMÁN WOLFHARD, L. V.; RAEDIG, C. Connectivity conservation management: linking private protected areas. In: NEHREN, U. et al. (ed.). Strategies and tools for a sustainable rural Rio de Janeiro. Cham: Springer International Publishing, 2019. p. 155-171. (Springer series on environmental management).

IGNATIEVA, M.; STEWART, G. H.; MEURK, C. Planning and design of ecological networks in urban areas. Landscape and Ecological Engineering, New York, v. 7, n. 1, p. 17-25, jan. 2011.

INSTITUTO CHICO MENDES DE CONSERVAÇÃO DA BIODIVERSIDADE. Floresta Nacional de Ipanema. [S. l.], 2010. Disponível em: http://icmbio.gov.br/flonaipanema/. Acesso em: 18 maio 2017.

LANDIS, J. R.; KOCH, G. G. The measurement of observer agreement for categorial data. Biometrics, Washington, v. 33, p. 159-174, 1977.

LATAWIEC, A. E. et al. Creating space for large-scale restoration in tropical agricultural landscapes. Frontiers in Ecology and the Environment, Washington, v. 13, n. 4, p. 211-218, may 2015.

MARTENSEN, A. C.; PIMENTEL, R. G.; METZGER, J. P. Relative effects of fragment size and connectivity on bird community in the Atlantic Rain Forest: implications for conservation. Biological Conservation, Amsterdam, v. 141, n. 9, p. 2184-2192, sep. 2008.

MCGARIGAL, K. FRAGSTATS help. Amherst: [s. n.], 2015. Disponível em: https://www.umass. edu/landeco/research/fragstats/documents/fragstats.help.4.2.pdf. Acesso em:20 mar. 2019.

MELLO, K. de; TOPPA, R. H.; CARDOSO-LEITE, E. Priority areas for forest conservation in an urban landscape at the transition between atlantic forest and cerrado. Cerne, Lavras, v. 22, n. 3, p. 277-288, jul./sep. 2016.

MENESES, P. R.; ALMEIDA, T. de (org.). Introdução ao processamento de imagens de sensoriamento remoto. Brasília, DF: UNB, 2012. 266 p.

PEREIRA, B. A. da S.; VENTUROLI, F.; CARVALHO, F. A. Florestas estacionais no cerrado: uma visão geral. Pesquisa Agropecuária Tropical, Goiânia, v. 41, n. 3, jul. 2011.

REZENDE, C. L. et al. From hotspot to hopespot: an opportunity for the Brazilian Atlantic Forest. Perspectives in Ecology and Conservation, Rio de Janeiro, v. 16, n. 4, p. 208-214, oct./dec. 2018. 
RIBEIRO, M. C. et al. The Brazilian Atlantic Forest: how much is left, and how is the remaining forest distributed? Implications for conservation. Biological Conservation, Amsterdam, v. 142, n. 6, p. 1141-1153, jun. 2009.

RODRIGUES, R. R.; BONONI, V. L. R. (ed.). Diretrizes para conservação e restauração da biodiversidade no Estado de São Paulo. São Paulo: Instituto de Botânica, 2008.

SÃO PAULO. APA Itupararanga. São Paulo: Sistema Ambiental Paulista, 2003. Disponível em: http://ambiente.sp.gov.br/apa-itupararanga/. Acesso em: 13 maio 2017.

SCHALKOFF, R. Pattern recognition: statistical, structural and neural approaches. New York: Wiley, 1992.

SEKERCIOGLU, C. H. Tropical ecology: riparian corridors connect fragmented forest bird populations. Current Biology, Cambridge, v. 19, n. 5, p. R210-R213, mar. 2009.

SILVA, M. S. F.; SOUZA, R. M. Padrões espaciais de fragmentação florestal na flona do Ibura Sergipe. Mercator, Fortaleza, v. 13, n. 3, p. 121-137, set./dez. 2014.

SISTEMA INTEGRADO DE GERENCIAMENTO DE RECURSOS HÍDRICOS DO ESTADO DE SÃO PAULO. Apresentação. São Paulo, 2018. Disponível em: http://sigrh.sp.gov.br/cbhsmt/ apresentacao. Acesso em: 20 mar. 2019.

SMITH, W. S. et al. Urban biodiversity: how the city can do its management? Biodiversity International Journal, Oklahoma, v. 2, n. 3, p. 246-251, may 2018.

TAMBOSI, L. R. Análise da paisagem no entorno de três unidades de conservação: subsídios para a criação da zona de amortecimento. 2008. Dissertação (Mestrado em Ciências) - Instituto de Biociências, Universidade de São Paulo, São Paulo, 2008.

TANNIER, C. et al. Impact of urban developments on the functional connectivity of forested habitats: a joint contribution of advanced urban models and landscape graphs. Land Use Policy, Enschede, v. 52, p. 76-91, mar. 2016.

UN. Department of Economic and Social Affairs. 2018 revision of world urbanization prospects. London, 2018. Disponível em: http:// un.org/development/desa/publications/2018-revision-ofworld-urbanization-prospects.html. Acesso em: 2 ago. 2018.

VALENTE, R. A.; PETEAN, F. C. de S.; VETTORAZZI, C. A. Multicriteria decision analysis for prioritizing areas for forest restoration. Cerne, Lavras, v. 23, n. 1, p. 53-60, jan./mar. 2017.

ZHANG, Z. et al. Enhancing landscape connectivity through multifunctional green infrastructure corridor modeling and design. Urban Forestry \& Urban Greening, Jena, v. 38, p. 305-317, feb. 2019. 